\title{
Comitês de ética em pesquisa no Brasil
}

\author{
Maiana de Oliveira Cerqueira e Costa ${ }^{1}$ \\ Pablo Ivan Pereira Ramos ${ }^{1}$ \\ Izolda Nunes Guimarães ${ }^{2}$ \\ Lilia Maria de Azevedo Moreira ${ }^{22}$
}

\begin{abstract}
Resumo
Os Comitês de Ética em Pesquisa (CEPs) se apresentam como ferramentas essenciais na busca pela eticidade nas pesquisas clínicas. A obrigatoriedade da submissão de projetos que envolvem seres humanos a um CEP foi somente instituída com a Resolução 196/96, de 1996, considerada um grande avanço na questão da ética em pesquisa no Brasil. O presente estudo teve como objetivo, através de um levantamento bibliográfico, analisar a implementação dos Comitês de Ética no Brasil, com ênfase no estado da Bahia. Verificou-se um aumento no número de Comitês de Ética, o que indica uma busca de adequação às novas diretrizes apresentadas pelo Conselho Nacional de Saúde, tanto por parte das instituições como dos pesquisadores. Observa-se, entretanto, que a distribuição desses Comitês é desigual, com maior concentração nas regiôes Sul e Sudeste do país.
\end{abstract}

Palavras-chave: Bioética; Comitês de Ética em Pesquisa- Brasil; Conselho Nacional de Saúde(Brasil)- Resolução 196/96.

\section{INTRODUÇÃO}

Os comitês de ética em pesquisa (CEPs) são instrumentos importantes na avaliação dos aspectos éticos que concernem à pesquisa com seres humanos. Sua composição é heterogênea, pois são formados por membros de diversas áreas de pesquisa e segmentos sociais, respeitando-se, portanto, diferentes pontos de vista, em prol da defesa dos interesses dos sujeitos da pesquisa (CONSELHO NACIONAL DE SAÚDE, 2002). Esses comitês surgiram, em todo o mundo, como resposta à demanda cada vez mais urgente pelo respeito aos aspectos éticos na pesquisa que envolve seres humanos, de maneira a se evitar a repetição de casos trágicos, como o Estudo Tuskegee, no Alabama, e as atrocidades cometidas pelos nazistas durante a Segunda Guerra Mundial. À época, tais experimentos não eram passíveis de punições nem considerados ilegais, apesar de já terem sua eticidade posta em questão. Nesse contexto de total desrespeito à dignidade humana, surge $\mathrm{o}$ Código de Nuremberg, em 1947, como um conjunto de princípios éticos a serem seguidos na experimentação que envolvesse pessoas, ao qual foram adicionadas normas complementares, quando do encontro da Associação Médica Mundial, em 1964, o que levou à gênese da Declaração de Helsinque, hoje consagrada em todo o mundo.

\footnotetext{
Correspondência para / Correspondence to:

Lilia Maria de Azevedo Moreira.

Departamento de Biologia - Instituto de Biologia - UFBA.

R. Barão de Geremoabo, 147 - Ondina.

40.179-290 Salvador - Bahia - Brasil.

E-mail: lazevedo@ufba.br
}

${ }^{1}$ Graduandos em Ciências Biológicas- Instituto de Biologia - Universidade Federal da Bahia .

${ }^{2}$ Docentes da disciplina BIOA66 - Bioética - Instituto de Biologia - UFBA 
A partir do Código de Nuremberg e da Declaração de Helsinque, diversos países, entre eles o Brasil, estabeleceram normas que consolidaram os princípios propostos nesses documentos em sua legislação no que tange à pesquisa com humanos. No Brasil, o Conselho Nacional de Saúde aprovou, em 1988, a Resolução 01/88, a qual dispõe, em seu artigo $4^{\circ}$ : "Em toda a pesquisa em que o ser humano for submetido a estudo, deverá prevalecer o critério de respeito à sua dignidade e a proteção de seus direitos e bem-estar". Ela abrange questôes relacionadas ao estudo de novas terapias e drogas, além de já ressaltar a necessidade do estabelecimento de comitês de ética em pesquisa em instituições onde são realizadas pesquisas com seres humanos e a obrigação da aprovação por esses comitês de projetos que envolvem pessoas (CONSELHO NACIONAL DE SAÚDE, 1988). Contudo, além de não ocorrer adesão de pesquisadores e instituições às normas dispostas na Resolução 01/88, tampouco houve maneira de acompanhar o funcionamento dos comitês (FREITAS, 1998). Foi somente em 1996, a partir da Resolução 196/96, que a obrigatoriedade dos CEPs fez-se cumprir, com a avaliação dos aspectos éticos das pesquisas que envolvem seres humanos por um comitê constituído para tal fim (FONTELLES; CARVALHO; D'OLIVEIRA, 2007). Segundo o artigo $7^{\circ}$ da Resolução 196/96, "Toda pesquisa envolvendo seres humanos deverá ser submetida à apreciação de um Comitê de Ética em Pesquisa”. Esse artigo ainda dispõe que: "As instituições nas quais se realizam pesquisas envolvendo seres humanos deverão constituir um ou mais de um Comitê de Ética em Pesquisa (CEP), conforme suas necessidades" (CONSELHO NACIONAL DE SAÚDE, 1996). Sendo assim, com todas as diretrizes e normas regulamentadoras aprovadas pela Resolução 196/96, o que se buscou foi a preocupação com a eticidade das pesquisas que envolvem seres humanos, ainda que de maneira imposta.

Este trabalho teve por objetivos: $1^{\circ}$.) o levantamento dos CEPs existentes no país, a partir de dados da literatura e do Ministério da Saúde; $2^{\circ}$.) a discriminação destes Comitês por estado e por instituições de ensino superior; $3^{\circ}$.) avaliar, em específico, os CEPs existentes no estado da Bahia e a relevância desse estado no plano nacional.

\section{OS COMITÊS DE ÉTICA EM PESQUISA}

De acordo com a Resolução 196/96, é atribuída aos CEPs a responsabilidade de revisar todos os protocolos de pesquisa que envolve seres humanos de maneira direta ou indireta, no sentido de avaliar a eticidade da pesquisa a ser realizada, que deve seguir os seguintes princípios: $1^{\circ}$.) autonomia, pois os sujeitos da pesquisa devem estar cientes de todos os procedimentos que serão adotados no decorrer da pesquisa com todos os possíveis riscos e danos através da assinatura do termo de consentimento livre e esclarecido, e a proteção a grupos vulneráveis e aos legalmente incapazes; $2^{\circ}$.) beneficência, buscando-se a maximização dos benefícios e a redução de danos e riscos; 3º.) não-maleficência, pela garantia de que danos previsíveis serão evitados; 40.) justiça e equidade, já que as pesquisas têm de ser relevantes, com vantagens significativas para os sujeitos e minimização dos ônus para aqueles vulneráveis. Também é função do CEP a elaboração de um parecer consubstanciado por escrito, que indique a decisão do Comitê em relação à aprovação ou não, e acompanhar o desenvolvimento dos projetos de pesquisa, caso sejam aprovados. Além disso, O CEP desempenha um papel educativo no sentido de promover a discussão e a reflexão sobre aspectos éticos na ciência, enfocando estudos que envolvem seres humanos.

Alguns protocolos de pesquisa em áreas temáticas especiais - como genética humana, reprodução humana, fármacos, medicamentos, vacinas e testes diagnósticos novos ou não registrados no país, equipamentos, insumos e dispositivos para a saúde novos ou não registrados no país, novos procedimentos ainda não consagrados na literatura, estudos com populações indígenas, projetos que envolvam aspectos de biossegurança, pesquisas coordenadas do exterior ou com participação estrangeira e pesquisas e projetos que envolvam remessa de material biológico para o exterior -, a critério 
do CEP, devem ser analisado por uma instância superior. Quando aprovados, devem ser encaminhados para a avaliação e aprovação pela Comissão Nacional de Ética em Pesquisa (CONEP), órgão ligado ao Ministério da Saúde. Tais áreas temáticas especiais necessitam de uma avaliação mais criteriosa dos aspectos éticos de pesquisas com seres humanos, por envolverem dilemas mais complexos. Portanto, projetos nessas áreas estão sujeitos à apreciação pelo CONEP, que desempenha o papel de coordenar a rede de CEPs, desenvolvendo também a regulamentação para a proteção dos sujeitos da pesquisa, incluindo a elaboração de normas específicas para as áreas temáticas. Para fortalecer o sistema CEPs-CONEP, está sendo instalado o Sistema Nacional de Informação sobre Ética (SISNEP), que objetiva otimizar a tramitação dos processos e facilitar aos pesquisadores o acompanhamento e a submissão dos projetos online. Os projetos aprovados em cada estado podem ser acessados por qualquer pessoa diretamente na página do SISNEP (http:// portal.saude.gov.br/sisnep/), o que permite maior transparência no acompanhamento dos resultados, embora não seja permitida a visualização dos métodos empregados, apenas os títulos dos projetos.

\section{COMITÊS DE ÉTICA EM PESQUISA NO BRASIL}

É notável o crescimento no número de CEPs registrados junto ao CONEP a partir da aprovação da Resolução 196/96, em 1996. Esse crescimento se deve, provavelmente, a uma busca pela adequação dos centros de pesquisa às novas diretrizes do Conselho Nacional de Saúde. Daí o incremento de 636\% no número desses Comitês em apenas 10 anos, conforme Tabela 1 e Figura 1.

De acordo com os dados recentes, dispostos na Tabela 1, são registrados, em média, 48 Comitês a cada ano, havendo um total acumulado de 534 CEPs cadastrados junto ao CONEP até maio de 2007. Para efeitos de comparação, até o ano 2000, no Reino Unido, país de excelência acadêmica reconhecida, eram registrados 20 Comitês de Ética (SLOWTHER;
Tabela 1. Comitês de ética em pesquisa (CEPs) registrados no CONEP.

\begin{tabular}{lcc}
\hline Ano & $\begin{array}{c}\text { Número de } \\
\text { CEPs regis- } \\
\text { trados no ano }\end{array}$ & $\begin{array}{c}\text { Número } \\
\text { acumulado de } \\
\text { CEPs registrados }\end{array}$ \\
\hline 1997 & 84 & 84 \\
1998 & 44 & 128 \\
1999 & 34 & 162 \\
2000 & 33 & 195 \\
2001 & 38 & 233 \\
2002 & 38 & 271 \\
2003 & 51 & 322 \\
2004 & 52 & 374 \\
2005 & 72 & 446 \\
2006 & 73 & 519 \\
2007 (1) & 15 & 534 \\
\hline
\end{tabular}

Fonte: Comissão Nacional de Ética em Pesquisa (CONEP); (1)Dados até maio de 2007.

Figura 1. Número de CEPs no Brasil entre 1997 e 2007 (até maio).

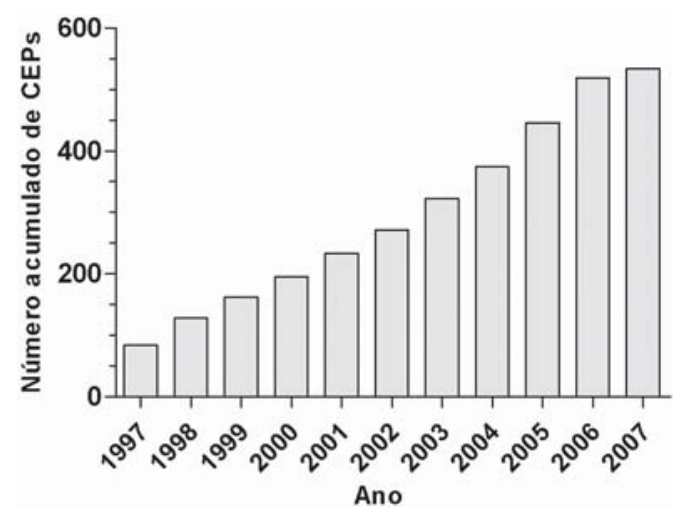

HOPE, 2000). Dividindo-se sua população pelo número de Comitês, resultaria em um para cada 2939000 habitantes, aproximadamente. Naquele mesmo ano, essa taxa, no Brasil, seria de um para cada 870764 habitantes. Atualmente, é de um para cada 346441 habitantes, o que coloca o Brasil numa posição comparável e até mesmo superior ao de países mais desenvolvidos quanto à questão da ética. Entretanto, ainda no Reino Unido, enquanto os projetos são apreciados pelas instâncias superiores em no máximo 60 dias, no Brasil, a apreciação pelo CONEP pode chegar a até um ano*, embora a Resolução 196/96, em

* Comunicado pessoal de pesquisador da UFBA que relatou atraso de cerca de um ano devido a demora na apreciação de projeto 
seu artigo VIII.4, letra 'c', indique como uma das atribuiçôes do CONEP a de "aprovar, no prazo de 60 dias, e acompanhar os protocolos de pesquisa em áreas temáticas especiais [...].” Por conta da morosidade e lentidão desses encaminhamentos, muitas vezes pesquisadores trabalham na clandestinidade - situação que, do ponto de vista bioético, é inaceitável. Jorge, Pegoraro e Ribeiro (2007), ao realizarem um levantamento dos projetos de um Hospital Universitário de Uberlândia, constataram que, enquanto $47,6 \%$ dos projetos que obtiveram fomento de órgão de apoio foram submetidos ao CEP da Instituição, apenas 7,1\% dos realizados sem esse apoio foram submetidos a essa apreciação. Em se tratando de um hospital, instituição em que a maioria senão a totalidade da pesquisa envolve seres humanos, todos os projetos deveriam ter sido submetidos ao Comitê de Ética em Pesquisa local. Os resultados desses autores, embora pontuais, sugerem a necessidade de uma reflexão crítica. Afinal, a nãoavaliação dos projetos que envolvem seres humanos pelos pares pode submeter os sujeitos da pesquisa a situaçôes de risco desnecessárias, que poderiam ser evitadas com o simples respeito aos princípios bioéticos já consagrados e dispostos na própria Resolução 196/96, entre eles o da ponderação entre riscos e benefícios para os sujeitos.

Dentre as Unidades Federativas, o estado de São Paulo possui mais CEPs (Figura 2), ao todo 159 , o que corresponde a $29,13 \%$ de todos os existentes no país. Em seguida, encontramse os estados de Minas Gerais (65 CEPs, 11,90\%), Rio de Janeiro (56 CEPs, 10,26\%), Rio Grande do Sul (42 CEPs, 7,69\%), Paraná (32 CEPs, 5,86\%) e Bahia (27 CEPs, 4,94\%). O predomínio do número de CEPs das regiōes Sul e Sudeste reflete a desigualdade na distribuição de recursos, o que acaba afetando a produção do conhecimento, que se concentra nas regiōes mais desenvolvidas economicamente, que detêm o maior número de Centros de Pesquisa. Um exemplo disso é o da regiāo Norte, que passa a ser representada pelo estado do Amazonas, em $11^{\circ}$ lugar, reflexo do distanciamento dessa região na produção de conhecimento. Em 2007, por exemplo, os
Figura 2. Número de CEPs, por estado, em instituições de ensino e não relacionadas, cadastradas junto ao CONEP até 2007.

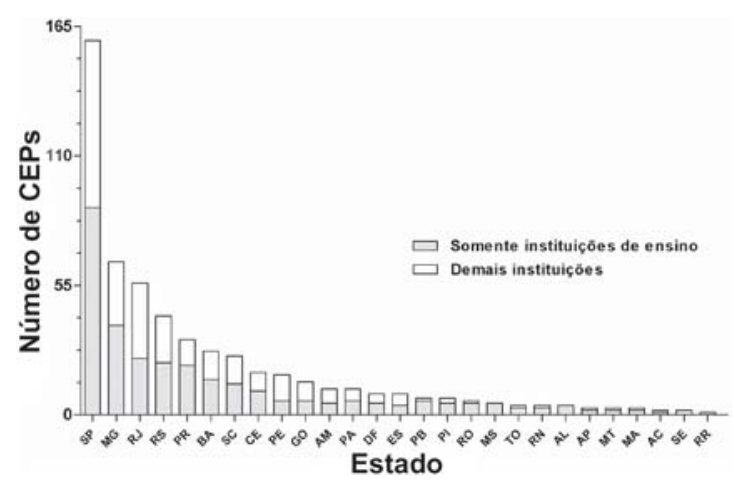

governos dos Estados do eixo Sul-Sudeste gastaram $85 \%$ de todos os recursos empregados em atividades científicas no país (BRASIL, 2008), o que reafirma o quadro exposto.

Foi também avaliado o quanto as Instituições de Ensino Superior (IES, Faculdades e Universidades) contribuem para o total de CEPs, já que é obrigatória a presença desses Comitês nas instituições que realizam pesquisas que envolvem seres humanos. A Figura 2 apresenta esses dados, separados por IES e por demais Instituições (e.g., hospitais e fundações particulares, desvinculadas do ensino superior). No cômputo geral, os Comitês de Ética em Pesquisa localizados em IES contribuem em 55\% do total de CEPs, somando 304 Comitês em todo o país.

\section{COMITÊS DE ÉTICA EM PESQUISA NA BAHIA}

$\mathrm{Na}$ Bahia, há um total de 27 CEPs, dos quais 15 localizam-se em faculdades e universidades (QUADRO 1), o que representa 55\% de todos os CEPs do estado. Um dos mais antigos, criado em 1997, localiza-se na Maternidade Climério de Oliveira, ligada a Universidade Federal da Bahia. O CEP dessa maternidade é o nono do país em número de projetos submetidos à apreciação pelo CONEP, condição imposta para projetos que pertencem às chamadas áreas temáticas especiais, incluindo reprodução 
Quadro 1. Comitês de Ética em Pesquisa (CEPs) de faculdades e universidades do estado da Bahia

\begin{tabular}{|lc|}
\hline CEP & Criação \\
\hline Maternidade Climério de Oliveira (UFBA) & 1997 \\
Hospital Universitário Prof. Edgar Santos (UFBA) & 1998 \\
Universidade Estadual de Santa Cruz (UESC) & 1999 \\
Instituto de Saúde Coletiva (UFBA) & 2001 \\
Universidade Estadual de Feira de Santana (UEFS) & 2002 \\
Escola Bahiana de Medicina & 2003 \\
Faculdade Adventista de Fisioterapia (FAFIS) & 2003 \\
Faculdade São Francisco de Barreiras & 2004 \\
Universidade Estadual do Sudoeste da Bahia (UESB) & 2005 \\
Escola de Nutrição (UFBA) & 2006 \\
Faculdade de Filosofia e Ciências Humanas (UFBA) & 2006 \\
Faculdade de Odontologia (UFBA) & 2006 \\
União Metropolitana de Educação e Cultura (UNIME) & 2006 \\
Universidade do Estado da Bahia (UNEB) & 2006 \\
Sociedade Mantenedora de Educação Superior da Bahia & 2007 \\
\hline
\end{tabular}

Fonte: Comissão Nacional de Ética em Pesquisa (CONEP)

humana e genética humana (COMISSÃO NACIONAL DE ÉTICA EM PESQUISA, 2006). Apenas outra instituição da região Nordeste (Universidade Federal do Ceará) figura na lista dos 20 CEPs que mais submeteram projetos ao CONEP, grupo composto prioritariamente por centros do Sul-Sudeste, o que evidencia a importância regional desses dois centros de pesquisa. $\mathrm{O}$ estado da Bahia, através da Universidade Federal da Bahia, destaca-se, desse modo, no âmbito da pesquisa nacional, já que o número de projetos submetidos ao CONEP é uma evidência indireta da atividade de pesquisa desenvolvida nas entidades.

Deve ser ressaltada a importância dos trabalhos desenvolvidos pelo Professor Elsimar Coutinho na Maternidade Climério de Oliveira, com investigação em biologia reprodutiva (também uma área temática especial), que implementou e desenvolveu seu núcleo de pesquisa a partir da década de 1980. Assim, o número de projetos submetidos ao CONEP oriundos dessa Instituição é também uma repercussão das atividades desse núcleo, ainda que não mais liderado por Coutinho. Além disso, a Maternidade é considerada o primeiro centro de referência do país para estudos e pesquisas na área da Reprodução Humana da
Organização Mundial da Saúde (UNIVERSIDADE FEDERAL DA BAHIA, [2008?] ).

\section{PROJETOS DE PESQUISA EM ÁREAS TEMÁTICAS ESPECIAIS}

No período de 1996 a 2004, verificou-se um incremento no número de projetos, em áreas temáticas especiais, submetidos ao CONEP. Tomando-se como base o relatório produzido no ano de 2004, foram emitidos por esse órgão 2650 pareceres para 1738 projetos submetidos à apreciação, sendo que mais da metade deles apresentaram pendências em sua metodologia e foram enviados aos pesquisadores para adequação às exigências do Comitê e novo reenvio (COMISSÃO NACIONAL DE ÉTICA EM PESQUISA, [2006] ).

Uma amostragem de 636 desses projetos (Figura 3) apresenta a área temática "cooperação estrangeira" como a que mais enviou projetos ao CONEP, 338 no total. Desses, $74 \%$ estavam associados à utilização de novos fármacos, o que indica o grande interesse de pesquisadores estrangeiros nessa área. Também nessa área foi encontrado o maior percentual de projetos 
Figura 3. Áreas temáticas especiais: número de projetos submetidos à apreciação do CONEP em 2004.

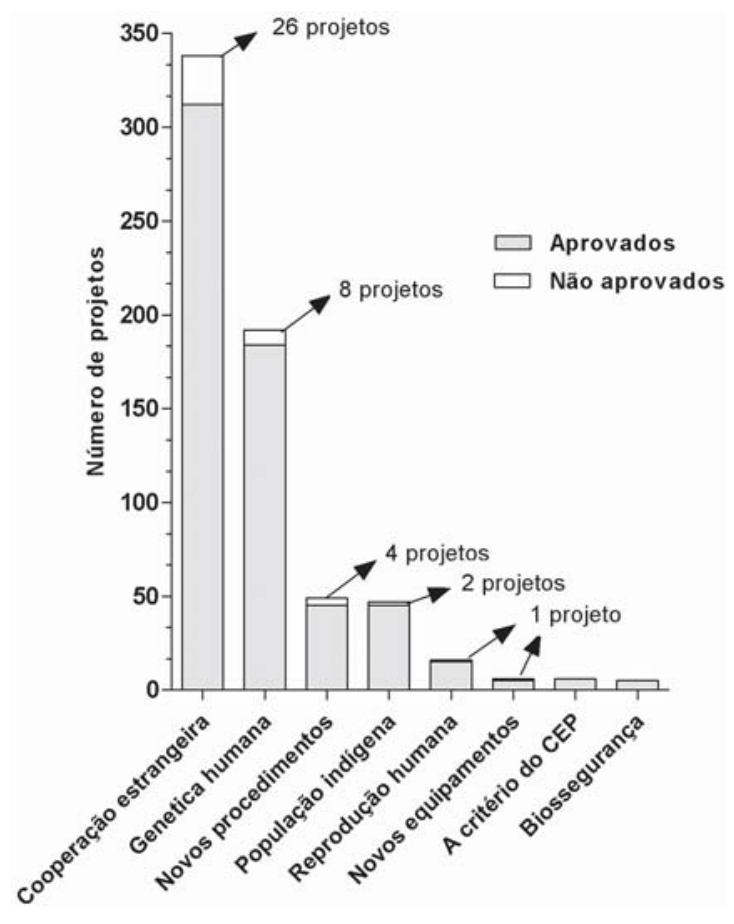

reprovados, $7,26 \%$ ao todo. Protocolos incompletos e Termos de Consentimento inadequados foram os principais motivos pelos quais os projetos foram considerados pendentes. As reprovações, contudo, não são frequentes, pois aos pesquisadores são concedidas oportunidades de correção do projeto e consequente adequação. Segundo Junges (2007), o Termo de Consentimento é uma peça fundamental na avaliação de um projeto de pesquisa por um Comitê de Ética. Daí a necessidade de o pesquisador debruçar-se sobre esse documento e redigi-lo de modo a ser o mais claro (em especial para o sujeito participante) quanto for possível.

As tendências expostas revelam lacunas nos protocolos em relação aos cuidados com os participantes das pesquisas, e indicam a necessidade de uma maior atenção dos Comitês quanto à identificação de situações classificadas como inadequadas, em especial em projetos que envolvem a utilização de novos fármacos, haja vista que eles podem produzir danos potenciais maiores aos sujeitos por conta de efeitos adversos ainda não descobertos.

\section{CONSIDERAÇÕES FINAIS}

Diante do exposto, fica evidente o aumento no número de Comitês de Ética em Pesquisa registrados junto ao CONEP a partir da Resolução 196/96, em 1996. Com isso, há uma busca pela adequação, tanto das Instituições quanto dos pesquisadores, às novas diretrizes apresentadas pelo Conselho Nacional de Saúde. Entretanto, a distribuição desses Comitês ainda é desigual, havendo uma concentração nas regióes Sul e Sudeste, evidenciando o 'abismo científico’ que ainda há entre essas regiões e o restante do país.

Entre os vinte CEPs que mais submeteram projetos ao CONEP, apenas dois estão localizados no Nordeste, um deles na Bahia e outro no Ceará, o que demonstra o predomínio absoluto das regiōes Sul e Sudeste e faz relembrar a política do "cafécom-leite", dessa vez na pesquisa científica. Contudo, a presença desses dois estados nordestinos já evidencia uma tentativa de romper a hegemonia das regiōes mais desenvolvidas na pesquisa cientifica, o que é um aspecto positivo.

Conclui-se que, embora a Resolução 196/ 96 tenha trazido avanços significativos na ética da pesquisa com seres humanos no Brasil, nem todas as instituições possuem um Comitê de Ética formado, e, muitas vezes, pesquisadores sequer submetem seus projetos a esses Comitês, trabalhando na clandestinidade e atribuindo esse procedimento à morosidade na avaliação dos projetos.

\section{Research ethics committees in Brazil}

\section{Abstract}

Research ethics committees (REC) are considered essential tools in the search for ethics in clinical research. 
The requirement of submission of projects involving human beings to a REC was only introduced with the Resolution 196/96, 1996, considered a major guideline on the issue of ethics in research in Brazil. This study aims, through a literature survey, analyzing the expansion of the Ethics Committees in Brazil, with emphasis on the state of Bahia. There was an increase in the number of ethics committees which indicates a search for adaptation to new guidelines presented by the National Board of Health, both by the institutions, as well as researchers. It is observed however that the distribution of these committees is uneven, with greater concentration in Southern and Southeastern regions of the country.

Keywords: Bioethics; Research Ethics Committees - Brazil; National Board of Health (Brazil)-Resolution $196 / 96$.

\section{REFERÊNCIAS}

BRASIL. Ministério da Ciência e Tecnologia. Investimentos nacionais em Ciência e Tecnologia (C\&T): Investimentos dos governos estaduais em ciência e tecnologia (C\&T), por região e modalidade 2000-2007. 2008. Disponível em: <http://www.mct.gov.br/ index.php/content/view/8838.html>. Acesso em: dez. 2008.

COMISSÃO NACIONAL DE ÉTICA EM PESQUISA (Brasil). Sistema CEPs - CONEP - 9 anos (1996 a 2005). [Brasília, DF, 2006]. Disponível em: <http://conselho.saude.gov.br/ comissao/conep/relatorio.doc $>$. Acesso em: nov. 2008.

CONSELHO NACIONAL DE SAÚDE (Brasil). Manual operacional para comitês de ética em pesquisa. Brasília, DF, 2002. Disponível em: <http://conselho.saude.gov.br/biblioteca/ livros/Manual_ceps.pdf>. Acesso em: nov. 2008.

CONSELHO NACIONAL DE SAÚDE (Brasil). Resolução $\mathbf{n}^{\circ} 001$, de 1988. Regulamenta o credenciamento de Centros de Pesquisa no país e recomenda a criação de um Comitê de Ética em Pesquisa (CEP) em cada centro. 1988. Disponível em: <http:// conselho.saude.gov.br/resolucoes/1988/ Reso01.doc>. Acesso em: nov. 2008.
CONSELHO NACIONAL DE SAÚDE (Brasil). Resolução no 196, de 10 de outubro de 1996. Aprova diretrizes e normas regulamentadoras de pesquisas envolvendo seres humanos. 1996. Disponível em: <http:// conselho.saude.gov.br/resolucoes/1996/ Reso196.doc>. Acesso em: nov. 2008.

FONTELLES, M.J.; CARVALHO, R.M.; D’OLIVEIRA, M.S. Estudo analítico do comitê de ética em pesquisa da Universidade da Amazônia. R. Paraense Med., Belém, v.21, n.2, p.19-22, 2007.

FREITAS, C.B.D. Os Comitês de Ética em Pesquisa: evolução e regulamentação. Bioética, Brasília, DF, v.6, n.2, 1998.

JORGE, M.T.; PEGORARO, B.L.; RIBEIRO, L.A. Abrangência de ação do Comitê de Ética em Pesquisa da Universidade Federal de Uberlândia. Bioética, Brasília, DF, v.15, n.2, p.308-316, 2007.

JUNGES, J.R. Exigências éticas do consentimento informado. Bioética, Brasília, DF, v.15, n.1, p.77-82, 2007.

SLOWTHER, A.M.; HOPE, T. Clinical ethics committees. BMJ, London, v.321, p.649-650, 2000.

UNIVERSIDADE FEDERAL DA BAHIA. Maternidade Climério de Oliveira. [2008?]. Disponível em: <http://portal.mec.gov.br/sesu/ arquivos/pdf/huclime.pdf>. Acesso em: nov. 2008. 